\title{
Evaluation de l'impact environnemental de la réutilisation des eaux usées épurées en irrigation : analyse ionique des sols
}

\author{
Hind MOUHANNI ${ }^{1^{*}}$, Hassan HAMDI ${ }^{2}$, Abdelaziz BENDOU $^{1}$, Eric CAVALLI ${ }^{3}$, Lhoussine BENZINE ${ }^{4}$ \\ $\underline{1}^{*}$ Laboratoire d'Ingénierie d'Energie et d'Environnement, ENSA, Agadir \\ ${ }^{2}$ Laboratoire de Mécanique des Fluides et d'Energétique, FSSM Marrakech \\ ${ }^{3}$ SERAC, Université de Franche-Comté, France \\ ${ }^{4}$ Responsable environnement, Régie Autonome Multiservices d'Agadir (RAMSA) \\ hind.mouhanni@gmail.com
}

\begin{abstract}
RESUME
Les eaux usées traitées par la station d'épuration (STEP) M'Zar du grand Agadir- Maroc peuvent être utilisées pour l'irrigation (catégorie $A$ normes OMS). Le volume actuel est de $10000 \mathrm{~m}^{3} / \mathrm{j}$ et atteindra prochainement $50000 \mathrm{~m}^{3} / \mathrm{j}$. Cette production d'eaux usées épurées (EUE) permettra de combler entièrement les besoins en eaux d'irrigation des espaces verts du grand Agadir.

Cette étude concerne la faisabilité de la réutilisation des EUE de la STEP de M'Zar pour l'irrigation des gazons des golfs. Elle présente la planification, le protocole et les résultats des essais qui ont été menés pour évaluer les impacts environnementaux. Nous présentons ainsi les résultats du suivi des analyses physicochimiques des sols prélevés pendant les 60 premiers jours d'irrigation. Le suivi de la qualité des sols des parcelles du gazon irriguées par les EUE et les EP permettent de mettre en évidence une augmentation de la salinité des sols. Cette salinisation des sols ne dépend pas seulement de la qualité des eaux d'irrigation mais aussi de la concentration et du type des sels solubles contenus dans les sols avant irrigation. La qualité des sols doit donc être prise en considération lors de la réutilisation des EUE pour l'irragation.
\end{abstract}

Mots clés : Réutilisation /eaux usées épurées /irrigation /gazon des golfs /salinité /analyses ioniques/sol.

\section{INTRODUCTION}

La région d'Agadir est une région agricole qui se caractérise par un climat aride, des ressources en eau très limitées et des sols pauvres en éléments nutritifs. Le secteur agricole est le plus grand consommateur d'eau. De ce fait, l'utilisation des Eaux Usées Epurées (EUE) pour l'irrigation peut contribuer à préserver les ressources en eau de la région De plus, vu la richesse des EUE en éléments fertilisants, cette solution permettra le recyclage de ces éléments et la diminution de l'usage abusif des engrais.

Le potentiel actuel des eaux usées traitées par la STEP M'Zar du grand Agadir, susceptible d'être utilisé sans restriction pour l'irrigation (catégorie $A$ normes OMS), peut atteindre $50000 \mathrm{~m}^{3} / \mathrm{j}$. Une étude de faisabilité sur la réutilisation des EUE de la STEP de M'Zar a été lancée par la Régie Autonome Multiservices d'Agadir (RAMSA). La superficie totale des espaces verts (public, privé, terrains de golf) est estimée à 878 ha avec un besoin en eau d'irrigation à satisfaire de l'ordre de 8 millions $\mathrm{m}^{3} / \mathrm{an}$. Avec un débit journalier de $50000 \mathrm{~m}^{3} / \mathrm{j}$, les EUE de la STEP de M'zar combleront entièrement ce besoin. Les terrains des golfs occupent à eux seuls 268 ha de la superficie totale des espaces verts d'Agadir (30,5\%), avec une consommation d'eau estimée à $3216000 \mathrm{~m}^{3}$ /an. Cette étude s'intéresse donc à l'utilisation des EUE pour l'irrigation des golfs. Elle présente la planification, le protocole et les résultats préliminaires des essais qui sont menés pour évaluer les impacts environnementaux de cette utilisation. Une attention particulière est accordée au suivi et à la comparaison des résultats des analyses ioniques des sols des parcelles du gazon irriguées par les EUE et de celles irriguées par les eaux de la nappe phréatique

\section{MATERIELS ET METHODES}

\subsection{Site expérimental}

Les essais in situ sont réalisés sur le site de la STEP de M'zar du grand Agadir où deux zones de terrain ont été aménagées : une pour les essais utilisant les EUE de la STEP pour l'irrigation et l'autre pour l'utilisation des eaux de la nappe phréatique (eau de puits) pour l'irrigation.

\subsection{Planification des essais}

Afin d'étudier la faisabilité et évaluer l'impact de l'utilisation des EUE sur l'irrigation du gazon des golfs, 3 variétés de gazon des golfs à savoir le Penccross (V1),le Ray-grass anglais (V2), et le mélange de 60\% du 
Ray- grass anglais et du 40\% du fétuque rouge (V3) ont été utilisées sur 3 parcelles de terrain $\left(P_{1}, P_{2}, P_{3}\right)$. À des fins de comparaison, les mêmes essais sont reproduits dans les mêmes conditions en utilisant les eaux de la nappe phréatique (eau de puits). Chaque parcelle a une dimension de $25 \mathrm{~m}^{2}$ et est subdivisée en deux parcelles de $12,5 \mathrm{~m}^{2}$ afin d'assurer une répétition des conditions de chaque essai. La subdivision de chaque parcelle est assurée par la pose d'un isolant en plastique sur une profondeur de 0,5m afin d'empêcher l'infiltration entre les subdivisions. Chaque parcelle contient une couche de $20 \mathrm{~cm}$ de sol composé de $75 \%$ de la terre végétale et de $25 \%$ de sable, ainsi qu'un lysimètre sur ses deux coins opposés. Les lysimètres ont un volume de $1 \mathrm{~m}^{3}$ et reproduisent les conditions du sol et de la variété de gazon ensemencée dans la parcelle concernée ; ils sont conçus avec une bonne étanchéité permettant la récupération du lixiviat après l'irrigation.

\subsection{Caractéristiques des sols et des eaux d'irrigation}

Les sols des parcelles sont composés de $75 \%$ de terre végétale et $25 \%$ de sable. Les analyses pédologiques des constituants du sol sont présentées dans le tableau 1.

Tableau 1. Résultats des analyses de la terre végétale et du sable.

\begin{tabular}{|l|c|c|c|c|c|c|c|c|c|}
\hline Paramètres & texture & $\mathrm{pH}$ & $\begin{array}{c}\text { Matière } \\
\text { organique }\end{array}$ & $\begin{array}{c}\text { Azote } \\
\text { total }\end{array}$ & $\begin{array}{c}\text { calcaire } \\
\text { total. }\end{array}$ & EC $1 / 5$ & $\begin{array}{c}\text { sels } \\
\text { soluble }\end{array}$ & $\begin{array}{c}\mathrm{P} 2 \mathrm{O} \\
\text { assimilable }\end{array}$ & $\begin{array}{c}\mathrm{K} 2 \mathrm{O} \\
\text { échangeable }\end{array}$ \\
\hline Unité & & & $\%$ & $\%$ & $\%$ & $\mathrm{dS} / \mathrm{m}$ & $\mathrm{g} / \mathrm{kg}$ & $\mathrm{ppm}$ & $\mathrm{ppm}$ \\
\hline T. végétale & Lsa & $\mathbf{8 , 7 0}$ & $\mathbf{1 , 8 5}$ & $\mathbf{0 , 1 5}$ & $\mathbf{5 , 2 0}$ & $\mathbf{0 , 1 2}$ & $\mathbf{0 , 4 1}$ & $\mathbf{1 4 , 5 6}$ & $\mathbf{1 2 8 , 7 0}$ \\
\hline Sable & $\mathbf{S}$ & $\mathbf{9 , 6}$ & $\mathbf{0 , 0 5}$ & $\mathbf{0 , 0 1}$ & $\mathbf{3 6 , 7}$ & $\mathbf{0 , 0 4 5}$ & $\mathbf{0 , 1 6}$ & $\mathbf{1}$ & $\mathbf{2 4 , 9}$ \\
\hline
\end{tabular}

D'après ces résultats, la terre présente une texture limoneuse avec peu d'argile et peu de sable. Elle est assez pauvre en éléments nutritifs majeurs (azote total, phosphore assimilable et potasse échangeable) et en matière organique. En outre, les sables sont très pauvres en tout élément fertilisant. La salinité des deux constituants du sol est très faible. L'alcalinité des sables est plus élevée que celle de la terre végétale. Donc le sol est propre à la culture mais il ne fournit aucun élément nutritif, ce qui impose d'apporter des éléments fertilisants par les eaux d'irrigation ou par d'éventuels amendements.

Les eaux utilisées pour l'irrigation des parcelles expérimentales sont de deux types :

- les eaux de la nappe phréatique de la plaine du Souss, pompées à partir des puits de surface de la station.

- les EUE de la STEP de M'zar du grand Agadir, traitées par le procédé d'épuration par infiltration percolation sur des lits de sable.

Les principales caractéristiques des eaux utilisées pour l'irrigation sont indiquées dans le tableau 2.

Tableau 2 : Résultats du bilan ionique des EUE et EP utilisées pour l'irrigation.

\begin{tabular}{|l|c|c|c|}
\hline \multicolumn{1}{|c|}{ Paramètres } & Unités & Eau puits (EP) & EUE \\
\hline Conductivité électrique (E.C) à $25^{\circ} \mathrm{C}$ & $\mathrm{dS} / \mathrm{m}$ & 7,4 & $\mathbf{7 , 1}$ \\
\hline Chlorure $\mathrm{Cl}^{-}$ & mole/l $\left(\times 10^{-3}\right)$ & $\mathbf{0 , 5 8}$ & $\mathbf{3 , 1 5}$ \\
\hline Potassium $\mathrm{K}^{+}$ & mole/l $\left(\times 10^{-3}\right)$ & $\mathbf{3 , 6 1}$ & $\mathbf{2 0 , 3 1}$ \\
\hline Sodium $\mathrm{Na}^{+}$ & mole/l $\left(\times 10^{-3}\right)$ & $\mathbf{1 , 3 2}$ & $\mathbf{2 1 , 2 1}$ \\
\hline Calcium $\mathrm{Ca}^{++}$ & mole/l $\left(\times 10^{-3}\right)$ & $\mathbf{1 , 1 9}$ & $\mathbf{2 , 3 1}$ \\
\hline Magnésium $\mathrm{Mg}^{++}$ & mole/l $\left(\times 10^{-3}\right)$ & $\mathbf{1 , 3 2}$ & $\mathbf{0 , 2 8}$ \\
\hline Phosphore & mole/l $\left(\times 10^{-3}\right)$ & $\mathbf{0 , 0 1}$ & $\mathbf{5 , 1 9}$ \\
\hline Bicarbonate $\mathrm{HCO}_{3}^{-}$ & mole/l $\left(\times 10^{-3}\right)$ & $\mathbf{3 , 1 3}$ & $\mathbf{0 , 8 8}$ \\
\hline Sulfate $\mathrm{SO}_{4}^{--}$ & mole/l $\left(\times 10^{-3}\right)$ & $\mathbf{0 , 1 0}$ & $\mathbf{5 , 6 4}$ \\
\hline Nitrates $\left({\left.\mathrm{N}-\mathrm{NO}_{3}\right)}\right.$ & mole/l $\left(\times 10^{-3}\right)$ & $\mathbf{0 , 0 8}$ & $\mathbf{6 , 6 6}$ \\
\hline Sodium Adsorption Ratio (SAR) & & $\mathbf{0 , 0 2}$ & \\
\hline
\end{tabular}

Les eaux de la nappe et celles épurées peuvent être classées comme eau d'irrigation selon l'USDA (United States Department of Agriculture). La classification USDA est basée sur les valeurs de la conductivité électrique (EC) et celle de l'indice SAR (Sodium Adsorption Ratio) [3]:

- Les eaux de la nappe sont classées C2S1, elles peuvent être utilisées sur n'importe quel type de sol. La salinité des eaux $(E C=0,520 \mathrm{ds} / \mathrm{m})$ est proche de la limite pour la croissance du gazon sans appliquer des traitements spéciaux pour la salinité (limite située à $E C=0,750 \mathrm{ds} / \mathrm{m}$ ). 
- Les EUE sont classées C4S1, elles sont très salines (valeur de EC à $25^{\circ} \mathrm{C}$ égale à 3,15 $\mathrm{ds} / \mathrm{m}$ ).Cependant, elles peuvent être appliquées sur n'importe quel type de sol vu la valeur du SAR< 10.

On constate que les EUE ont une charge ionique importante vu leurs teneurs en chlorure, sodium et nitrates, pouvant affecter l'absorption d'autres cations (ex magnésium). En revanche, d'autres éléments minéraux indispensables pour la croissance du gazon dépassent les besoins comme le calcium qui contribue à alimenter le complexe d'échange du sol [3].

La comparaison de l'apport des eaux d'irrigation en éléments majeurs (N, P, K) est rapportée dans le tableau 3.

Tableau 3. Apport en NPK (kg/ha/an) par les eaux d'irrigation pour un volume de $1314 . \mathrm{m}^{3} / \mathrm{ha} / \mathrm{an}$.

\begin{tabular}{|c|c|c|c|}
\hline Eléments minéraux & Eau de la nappe & Eau usée épurée & Besoin théorique [1] \\
\hline $\mathbf{N}(\mathrm{kg} / \mathrm{ha} / \mathrm{an})$ & 6,83 & 404 & 300 \\
\hline $\mathbf{P}(\mathrm{kg} / \mathrm{ha} / \mathrm{an})$ & 0,000 & 11,6 & 100 \\
\hline $\mathbf{K}(\mathrm{kg} / \mathrm{ha} / \mathrm{an})$ & 0,092 & 56,5 & 200 \\
\hline
\end{tabular}

Les eaux de la nappe phréatique sont très pauvres en éléments nutritifs par rapport aux teneurs des EUE. Ces dernières sont très riches en azote et en potassium et pauvres en phosphore. II ressort de ces analyses que les EUE peuvent avoir des effets positifs sur la fertilité du sol et sur le rendement des cultures à condition qu'elles restent conformes aux recommandations OMS pour l'irrigation des cultures (catégories A).

\subsection{Protocole d'irrigation de semences et de suivi}

Les parcelles irriguées par les EUE sont éloignées de celles irriguées par les eaux de puits pour éviter toute contamination. Leur irrigation est effectuée par le même système. II s'agit d'une aspersion par pistolet ayant un débit constant de $828 \mathrm{l} / \mathrm{h}$, ce qui permet la maîtrise du même volume d'eau d'irrigation. Chaque parcelle de 25 $\mathrm{m}^{2}$ est irriguée trois fois par jour et reçoit au total 90 litres d'eau par jour.

Un des objectifs de l'étude vise à comparer la germination de trois variétés du gazon des golfs utilisées dans la région. II s'agit du Penccross (V1), du Ray-grass anglais (V2), et du mélange de $60 \%$ du Ray- grass anglais et du 40\% du fétuque rouge (V3). Les trois variétés ont été ensemencées respectivement sur les parcelles ( $\mathrm{P} 1$, $\mathrm{P} 2$ et P3) et irriguées avec les EUE et celles de la nappe pour comparaison.

Le suivi a débuté en juin 2007 par une semence des graines qui a été effectuée à la main. Les premiers plants du gazon ont germé après trois jours de semis pour la variété V2 et cinq jours pour les variétés V1 et V3. Les prélèvements des échantillons composites du sol sont effectués tous les 6 jours à partir du premier jour de germination. Ce suivi a duré 60 jours au total résultant en 10 compagnes d'échantillonnage. Pour chaque compagne 6 échantillons du sol sont récoltés pour être analysés. Les échantillons concernent les $20 \mathrm{~cm}$ supérieurs du sol de chaque parcelle.

Le temps de germination pour les deux qualités d'eaux est le même ce qui prouve que les conditions initiales du sol et du semis sont pareilles pour les différentes parcelles.

\section{RESULTATS ET DISCUSSION}

Les analyses ioniques des sols irrigués par les deux types d'eau d'irrigation ont porté sur les paramètres suivants : $\mathrm{pH}$, conductivité électrique (EC), sodium, calcium, magnésium, chlorures, ratio d'adsorption du sodium (SAR), sulfates et bicarbonates (figures 1 et 2 ).

Dans tous les cas, on constate des valeurs du pH voisines de la neutralité : entre 7,1 et 8,8 avec une moyenne de 7,9. Le pH du sol est constant dans le temps indépendamment de la variété du gazon et du type d'eau d'irrigation.

Par contre, les fluctuations des courbes de la conductivité électrique (EC) présentent une différence de comportement selon le type et la salinité d'eau d'irrigation utilisée. Les sols du gazon irrigués par les eaux de puits présentent des valeurs de la CE qui varient entre 0,46 et 10,4 dS/m avec une moyenne de 4,07 dS/m. La valeur moyenne des concentrations en sels solubles est de 0,0034 $\mathrm{g} / \mathrm{kg}$ pour les trois variétés. Rappelons que l'EC et les sels solubles de la terre végétale (TV), du sable (S), et du composé ( $75 \%$ TV et $25 \%$ S) sont respectivement de 0,$12 ; 0,045 ; 0,09 \mathrm{dS} / \mathrm{m}$ et 0,$41 ; 0,16 ; 0,34 \mathrm{~g} / \mathrm{kg}$. Les sels solubles du composé de la (TV) et du (S) ont diminué de $99 \%$. Ceci est expliqué par une lixiviation des sels vers la nappe par les eaux d'irrigation. Pour les sols irrigués par les EUE, les valeurs de l'EC varient entre 1,4 et $59 \mathrm{dS} / \mathrm{m}$ avec une moyenne de 22,94 dS/m. La valeur moyenne des concentrations des sels solubles est de $0,016 \mathrm{~g} / \mathrm{kg}$ pour les trois variétés. Les sels solubles du composé ont diminué de $96 \%$ à cause de la lixiviation par les eaux épurées salines ce qui augmente aussi l'EC des sols et par la suite de la nappe phréatique [1]. 


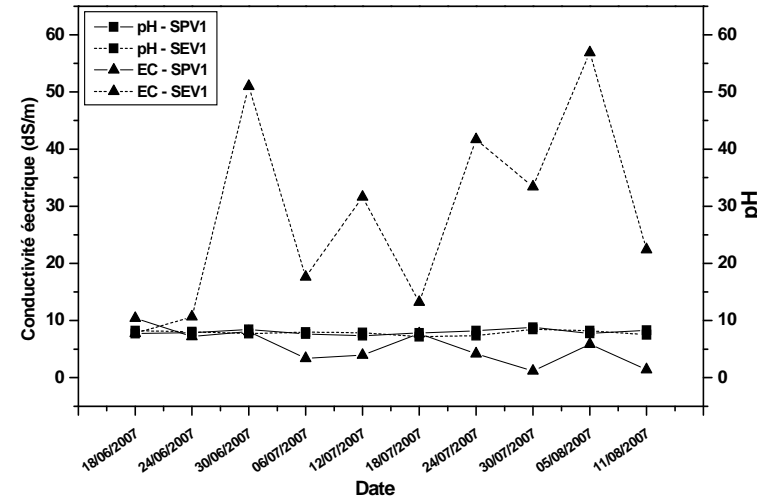

(a)

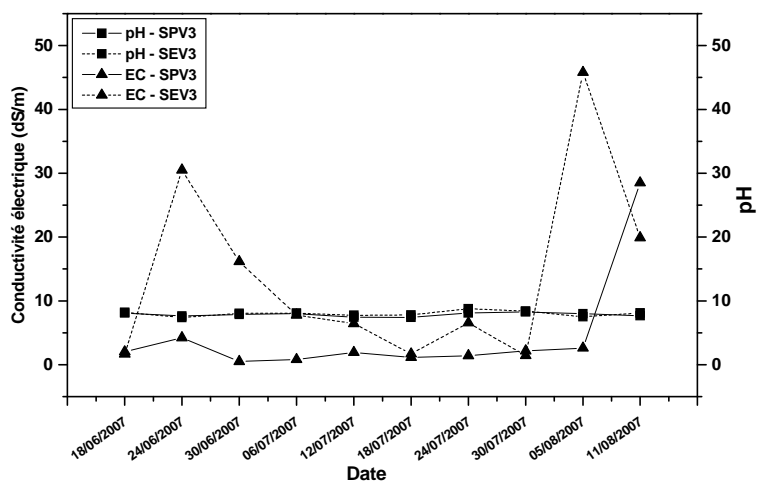

(c)

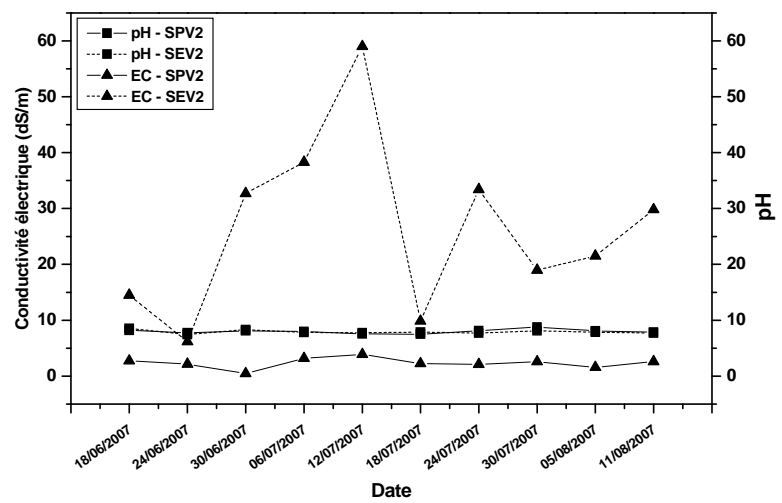

(b)

SEVi : sol des parcelles irriguées par les eaux épurées et contient la variété i

SPVi : sol des parcelles irriguées par les eaux de puits et contient la variété i

Figure 1 : Evolution du $\mathrm{pH}$ et de la conductivité électrique dans les sols des parcelles ensemencées par les trois variétés de gazon : (a) V1 ; (b) V2 ; (c) V3 irriguées avec les eaux puits et les eaux épurées.

La figure 2 montre que les sols irrigués par les eaux de puits ont des concentrations de $\mathrm{Na}+, \mathrm{Ca}++, \mathrm{Mg}++$ qui sont relativement constantes alors que la concentration du $\mathrm{Cl}$ - qui présente des fortes variations. Les concentrations moyennes du sodium, magnésium, calcium et du chlorure sont de 14,$9 ; 5,0 ; 7,4$ et 62,6 $\left(\times 10^{3}\right)$ mole/kg respectivement, le SAR est de 3,79 . On constate de fortes concentrations en chlorure des sodium : originellement présents dans le substrat ils sont dissous lors de l'irrigation [4].

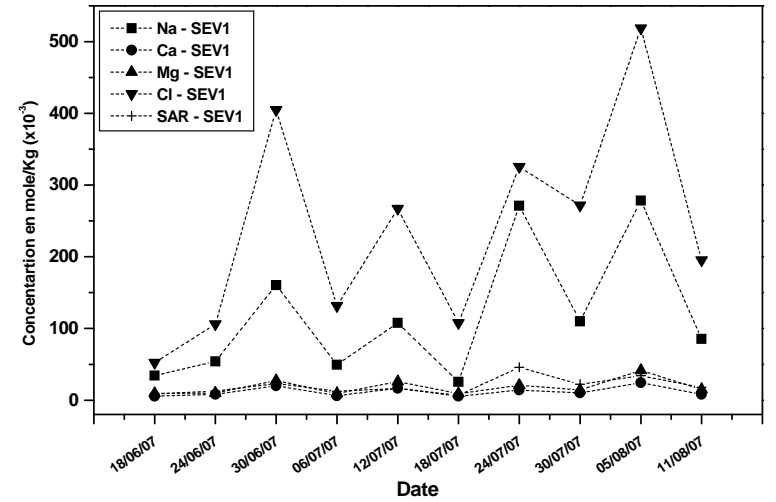

(a)

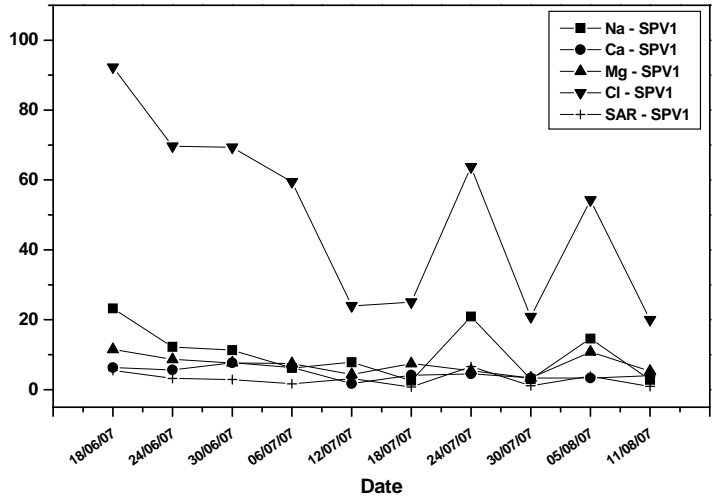

$\left(a^{\prime}\right)$ 


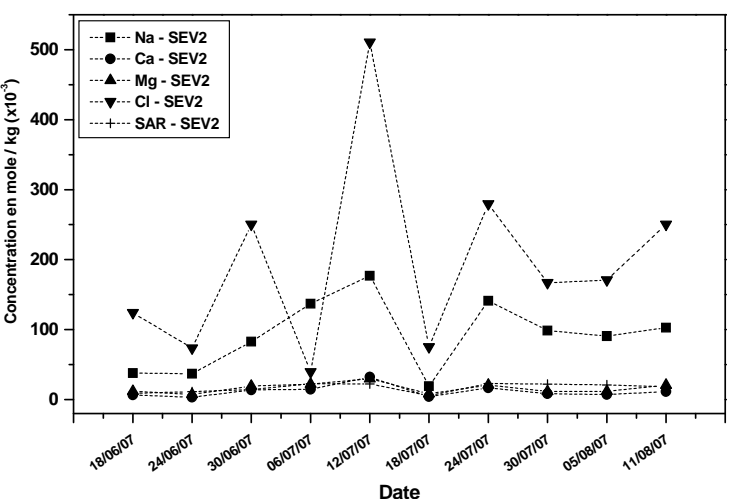

(b)

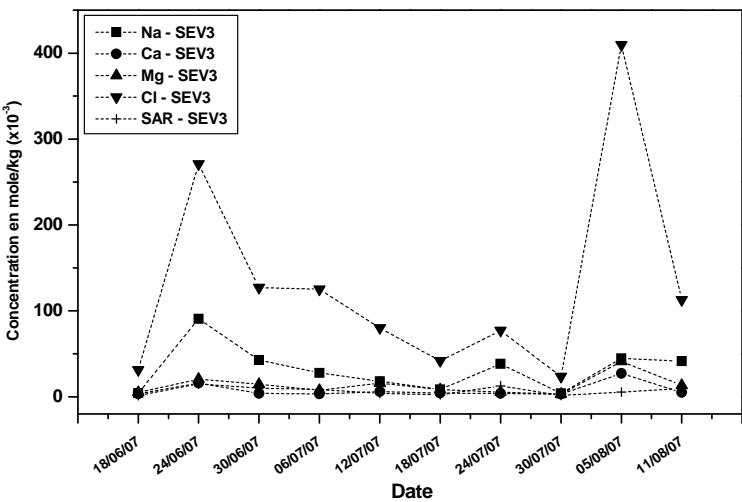

(c)

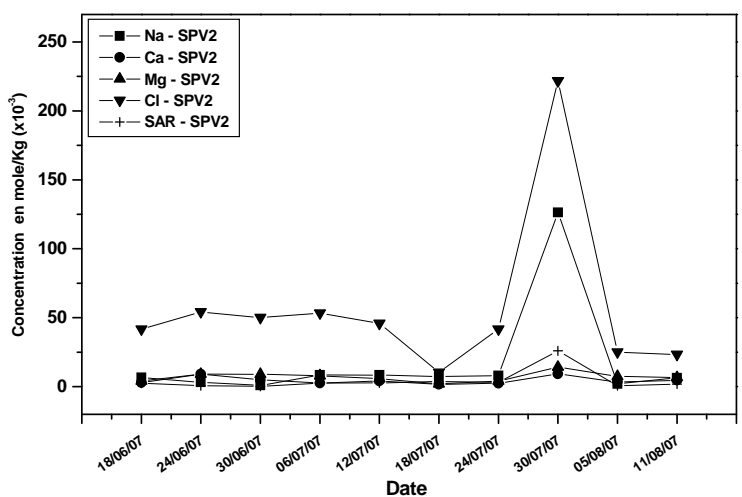

$\left(b^{\prime}\right)$

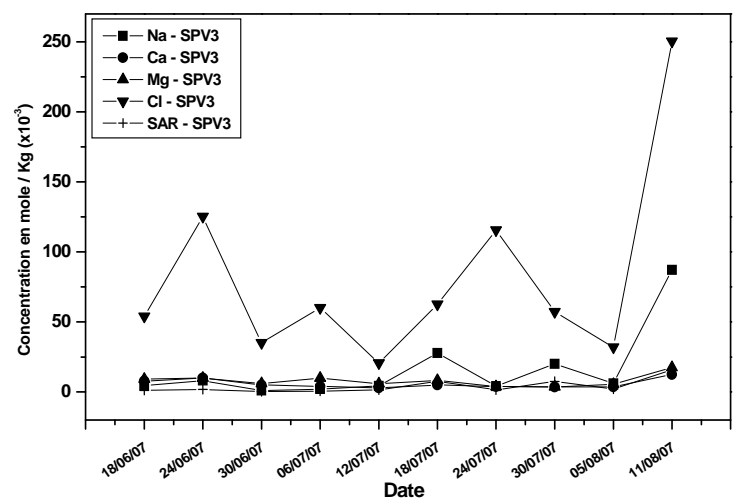

$\left(c^{\prime}\right)$

SEVi : sol du gazon irriguée par les eaux épurées et contient la variété i SPVi : sol du gazon irriguée par les eaux de puit et contient la variété i

Figure 2: Evolution du sodium; calcium; magnésium; chlorure et le SAR dans les sols des parcelles ensemencées par trois variétés du gazon : V1 (a) ; V2 (b) ; V3 (c) irrigués avec les eaux épurées et V1 (a') ; V2 (b') ; V3 (c') les eaux de puits.

L'apport de la salinité aux sols irrigués par les eaux de la nappe est très faible par rapport aux sels existant dans la terre végétale et le sable. Par contre l'utilisation des EUE comme eau d'irrigation s'accompagne d'une augmentation des niveaux de concentration des ions d'une manière plus importante que celles observées dans les sols irrigués par les eaux de puits.

Les concentrations moyennes du sodium, magnésium, calcium et du chlorure sont de 80,$7 ; 10,4 ; 16,01$; 14,64 et $187,36\left(\times 10^{3}\right)$ mole/kg respectivement et le SAR est de 14,64 .

L'accumulation des chlorures du sodium est rapider au début de l'irrigation. Elle est due à l'adsorption du chlorure de sodium sur les agrégats du sol. Le suivi des ions $\mathrm{Ca++}$ et $\mathrm{Mg}++$ ne présente aucun changement significatif après l'irrigation par les deux type d'eaux d'irrigation [2].

Les concentrations moyennes des sulfates et des bicarbonates dans les sols du gazon irrigués par les EUE et les EP sont de 7,$06 ; 6,5$ et 5,$54 ; 6,55\left(\times 10^{3}\right)$ mole/kg respectivement. Les concentrations des sulfates et des bicarbonates rapportent une constante dans le temps quelque soit le type de gazon et la qualité d'eau d'irrigation utilisé, malgré la formation des précipités de carbonate de calcium et de magnésium lors de l'irrigation par les EUE [3].

\section{CONCLUSION}

Le suivi de la qualité des sols des parcelles du gazon irriguées par les EUE et les EP nous ont permis de mettre en évidence une augmentation de la salinité des sols. Cette salinisation des sols ne dépend pas seulement de la qualité des eaux d'irrigation mais aussi de la concentration et du type des sels solubles 
contenus dans les sols avant irrigation. Les sels solubles sont lixiviés au cours de l'irrigation. La qualité des sols doit donc être prise en considération lors de la réutilisation des EUE pour l'irrigation.

\section{REMERCIEMENTS}

Nos remerciements s'adressent à la Régie Autonome Multiservice d'Agadir (RAMSA), l'Office Régionale de Mise en Valeur Agricole du Souss Massa Daraa (ORMVAS), I'Institut Nationale de Recherche Agronomique d'Agadir (INRA) et au laboratoire de chimie des eaux à Besançon en France (SERAC).

\section{RÉFÉRENCES BIBLIOGRAPHIQUES}

[1] F.Chenini, M.Trad, S.Réjeb, Z.Châabouni, D.Xanthoulis, (2002)."Optimisation et durabilité du traitement et de la réutilisation des eaux usées en agriculture", Rapport Ministère de l'agriculture, de l'environnement et des ressources hydrauliques, Institut National de Recherche en Génie Rural, Eaux et Forêts, Tunisie.

[2] M.Bazza, D.Xanthoulis, (2004)" Irrigation avec les eaux usées traitées ", manuel d'utilisation de la FAO.

[3] R.S.Ayers, D.Westcot (1988), " la qualité de l'eau en agriculture ", Bulletin FAO d'irrigation et du drainage.

[4] M.Jalali, (2008) "Effect of sodium and magnesium on Kinetics of potassium release in some calcareous soils of Western Iran", Geoderma, 145, 207-215. 